\title{
No excuse not to report complications using standardized methodology
}

\author{
Declan G. Murphy, MD; Marni Basto, MD
}

Division of Cancer Surgery, University of Melbourne, Peter MacCallum Cancer Centre; and Australian Prostate Cancer Research Centre, Epworth Richmond Hospital, Melbourne, Australia

See related article on page 179 .

Cite as: Can Urol Assoc J 2013;7(5-6):e445. http://dx.doi.org/10.5489/cuaj.1371

S tandardized reporting of postoperative complications using validated methodology is a highly desirable aim of any surgical audit process. In the past 10 years, the revised Clavien-Dindo Classification System (RCCS) has emerged as the most popular and best recognized system with which to grade complications occurring up to 90 days postoperatively. ${ }^{1}$ Although originally designed for a general surgery/hepato-biliary cohort, it has since been validated in other surgical specialties and is now in popular use. Indeed, many journals now insist on the use of this system when reporting surgical outcomes.

In an interesting paper in CUAJ, El Elkoushy and colleagues observed inter-rater variability in applying the RCCS to urological complications between post-graduate trainees (PGTs) $(n=16)$ and attending urologists $(n=16) .{ }^{2}$ Twenty cases were presented from an academic teaching centre with an overall good agreement rate of $81 \%$ and no statistical difference in overall RCCS grades between the groups. In their study, $65 \%$ of cases had an agreement rate above $80 \%$. Of the 7 cases with less than $80 \%$ agreement, 6 of these occurred in assigning grades to Clavien III and IV where subdivisions of classification may have contributed to ambiguity. Interestingly, there was slightly better intra-rater concordance within the PGT group (82\%) than the attending urologists (69\%). The overall conclusion from this study was that PGTs are equally able to complete Quality Assurance meeting reports using the RCCS at this hospital.

We, and others, have applied the RCCS to reporting of various urological procedures, including robotic radical prostatectomy and percutaneous nephrolithotomy and have encouraged the use of this system. ${ }^{3,4}$ However, the application of the RCCS to urological surgery is not without its limitations. As the current paper nicely illustrates, interobserver variability is an issue due to the subjective nature of the grading system, although there would appear to be some concordance across both trainees and specialists in this study. Also, as the system relies on the intervention required to manage a complication, there may be other complications (e.g., functional complications), which may not be adequately captured but which may have clinical importance. Nevertheless, it is only through the use of a standardized system, such as this, that outcomes can be improved by identifying and targeting areas in which adjustments to surgical practice or changes to perioperative pathways may lead to reduced postoperative morbidity. A standardized system is also essential to allow comparisons to be made between different surgical approaches to a particular procedure.

Interpretation of a complication and assignment of a grade would clearly be more straightforward if the RCCS was to be validated in a urological cohort and this would be a very welcome step. In the meantime, we should be reassured about the broad concordance reported in this paper when applying the RCCS to urological patients and should embrace the use of this standardized system as part of our audit and quality assurance processes in our daily practice.

Competing interests: None declared.

\section{References}

1. Dindo D, Demartines N, Clavien PA. Classification of surgical complications: a new proposal with evaluation in a cohort of 6336 patients and results of a survey. Ann Surg 2004;240:205-13. http://dx.doi.org/10.1097/01.sla.0000133083.54934.ae

2. Elkoushy MA, Luz MA, Benidir T, et al. Clavien classification in urology: Is there concordance among post-graduate trainees and attending urologists? Can Urol Assoc J 2013;7:179-84. http://dx.doi.org/10.5489/cuaj.505

3. Morgan $M$, Smith $N$, Thomas $K$, et al. Is Clavien the new standard for reporting urological complications? BJU Int 2009;104:434-6. http://dx.doi.org/10.1111/i.1464-410X.2009.08516.x

4. Murphy DG, Kerger M, Crowe $H$, et al. Operative Details and Oncological and Functional Outcome of Robotic-Assisted Laparoscopic Radical Prostatectomy: 400 Cases with a Minimum of 12 Months Follow-up. Eur Urol 2009;55:1358-67. http://dx.doi.org/10.1016/i.eururo.2008.12.035

Correspondence: Dr. Declan G Murphy, Division of Cancer Surgery, Peter MacCallum Cancer Centre, East Melbourne, VIC 3002, Australia; declan.murphy@petermac.org 